Guatemala, Guatemala City, Guatemala; ${ }^{5}$ Tephinet Inc., University of North Carolina, Del Valle University of Guatemala, Guatemala City, Guatemala

Background Men who have sex with men (MSM) and transgender persons are disproportionately affected by sexually transmitted infections (STIs), including HIV, in Guatemala. Access to integrated sexual health prevention and treatment services is limited. The purpose of this study was to identify barriers to accessing sexual health services among gay, bisexual, and non-gay identified MSM and male-to-female transgender persons in Guatemala City to inform the development of high quality and population-friendly services that are sensitive to the needs of this population.

Methods Semi-structured in-depth interviews were conducted with 27 purposively sampled participants, including 7 transgender, 11 gay, 5 bisexual, and 4 non-gay-identified participants, in Guatemala City. Interview topics included experiences with sexual health services, perceived barriers to access, social and sexual network characteristics, and HIV risk behaviours. Topical codes were developed based on readings of interview transcripts and codes were applied to the data using the qualitative software Atlas.ti. Data were compared between study sub-groups using thematic matrices and analytic memos.

Results Across all participants, public clinics were the most commonly used sexual health services due to their lower cost and greater accessibility, but many participants provided examples of discrimination, violation of confidentiality, and distrust in the quality of services offered. Transgender and gay participants preferred clinics where they felt a sense of belonging while non-gay identified participants preferred clinics that were not associated with the MSM community. The most prominent barriers to sexual health services described by participants included fear of discrimination related to sexual identity and/or behaviour, fear of having HIV and the associated stigmatisation, cost, and lack of social support.

Conclusions Findings highlight the need to strengthen and expand existing public STI clinics to improve access to services among MSM and transgender populations in Guatemala City. These services must address the multiple layers of stigma and discrimination that MSM and transgender persons experience related to identity, behaviour, and STI/HIV. Insights from this study are currently being applied to the implementation of two public clinics in Guatemala City that seek to provide a discrete, non-judgemental environment where individuals can seek affordable services without fear.

\section{P5-S6.18 COST-EFFECTIVENESS OF INTRODUCING RAPID SYPHILIS TESTING IN THE AMAZON REGION, BRAZIL}

doi:10.1136/sextrans-2011-050108.574

${ }^{1} \mathrm{C}$ H Carvalho, ${ }^{1} \mathrm{~A}$ Benzaken, ${ }^{2} \mathrm{R}$ Peeling, ${ }^{2} \mathrm{~A}$ Santos, ${ }^{2} \mathrm{~F}$ Terris-Prestholt. ${ }^{1}$ Alfredo da Matta Foundation, Manaus, Brazil; ${ }^{2}$ London school of Hygiene and Tropical Medicine, London, UK

Background This study aims to estimate the costs-effectiveness of introducing a universal syphilis screening and treatment package with an enhanced quality assurance system among isolated indigenous populations in the Amazon region of Brazil.

Methods In three indigenous health units (Umariacu II, Vendaval and Betania), incremental financial and economic costs of the full programme were collected including start-up, training and quality assurance, supervision and implementation. These units were chosen because of their different geographic access levels (easy to very difficult) so that results can be extrapolated to other health districts of the Amazon state. Unit cost per person screened and treated was estimated as well as the cost-effectiveness per adverse outcome and DALY averted. This study also provides the first data on prevalence rates in this population.

Results Using rapid syphilis tests, the prevalence of syphilis was $1.56 \%$ and $2.2 \%$ in the sexually active population and in pregnant women respectively at the three health units. The total financial cost of syphilis screening 4173 people was $\$ 277853$. The total economic cost was $\$ 285995.67$. The economic cost per person screened was US\$68.53 and treated was US\$4028.11. The cost per DALY saved was US\$484.31 (including stillbirth). Personnel costs contributed the largest input category consisting of $87.5 \%$ of the total costs, due to the high cost of labour of FUNASA (National Health Foundation) personnel. Training costs are also high due to the frequency of staff turnover and thus the need for repeated trainings.

Conclusions Although the cost per person screened and per person treated for syphilis could be considered high by international standards, the only alternative to screening in the health units for this population would be transporting people to the nearest larger town for screening in the nearest health facility with a laboratory. This would clearly exceed the costs of treating the cases locally by far. This therefore makes rapid syphilis testing the most costefficient alternative for testing these remotely located populations. Additionally, because of cultural behaviour of the indigenous populations and the fact that some of them are located near border regions of Brazil, the prevalence scenario can change quickly, increasing the number of syphilis and HIV cases in the absence of prompt identification and treatment.

\section{P5-S6.19 PREVALENCE AND INCIDENCE OF SEXUALLY TRANSMITTED INFECTIONS AMONG FEMALE SEX WORKERS IN TWO CITIES IN INDIA: IMPLICATIONS FOR STI CONTROL STRATEGIES}

doi:10.1136/sextrans-2011-050108.575

${ }^{1} \mathrm{~A}$ Das, ${ }^{1} \mathrm{P}$ Prabhakar, ${ }^{1} \mathrm{P}$ Narayanan, ${ }^{1} \mathrm{G}$ Neilsen, ${ }^{1} \mathrm{G}$ Morineau, ${ }^{2} \mathrm{~S}$ Mehendale, ${ }^{2}$ A Risbud. ${ }^{1}$ FHI, New Delhi, India; ${ }^{2}$ National AIDS Research institute, India

Background India is a large country with marked heterogeneity in prevalence of sexually transmitted infections (STIs) which has implications for STI control strategies. The study objective was to measure the prevalence and incidence of common bacterial STIs in a cohort of female sex workers (FSWs) in known high STI prevalence cities in response to a package of standardised interventions under Avahan, the India AIDS Initiative of the Bill \& Melinda Gates Foundation.

Methods FSWs attending clinics were followed up periodically over 6-9 months. At every visit, vaginal swabs were tested for Neisseria gonorrhoeae (GC) and Chlamydia trachomatis (CT) by Gen-Probe APTIMA Combo II. During the baseline and final visits, vaginal swabs were tested for Trichomonas vaginalis (TV) by PCR and blood was tested for syphilis using Rapid Plasma Reagin (RPR) with confirmatory Treponema pallidum Haemagglutination Assay (TPHA). All participants received presumptive treatment for gonorrhoea and chlamydia at the baseline visit and syndromic STI management at all subsequent visits.

Results A total of 417 FSWs were recruited, 360 returned for at least one follow-up visit, and 282 completed the final visit. The total follow-up period was 109.4 person years (median 0.18 years, maximum 1.07 years). Self-reported consistent condom use with commercial and regular partners was $70 \%$ and $17 \%$, respectively. A substantial proportion of cervical and trichomonal infections were asymptomatic (see Abstract P5-S6.19 table 1). The incidence of GC/ CT and TV was 1.0 and 2.0 per person year respectively. Three new cases of latent syphilis were detected at the final visit. 
Abstract P5-S6.19 Table 1 Prevalence of STls and asymptomatic infections $(\mathrm{n}=282)$

\begin{tabular}{llll}
\hline Pathogen & Baseline visit & 1st follow-up & Final visit \\
\hline Cervical & $27.7 \%$ (asymptomatic & 16.7\% (asymptomatic & $21.3 \%$ (asymptomatic \\
infections & $35.9 \%$ ) & $60.5 \%$ ) & $76.7 \%$ ) \\
(GC and/or CT) & & & \\
TV & $29.9 \%$ (asymptomatic & & $46.6 \%$ (asymptomatic \\
& $27.2 \%$ ) & & $82.2 \%)$ \\
Latent & $6.8 \%$ & $2.5 \%$ \\
syphilis (RPR & & \\
titre $>1: 8$ ) & & \\
\hline
\end{tabular}

Conclusions The incidence of GC, CT and TV and the considerable burden of asymptomatic infections indicate the need for periodic presumptive treatment at high STI prevalence sites in India. The current regime of bi-annual syphilis screening is justified. Consistent condom use and partner treatment, particularly for regular partners, need to be re-emphasised.

\section{P5-S6.20 STIS AND HEALTHCARE REFORM. WILL IT REALLY IMPROVE AMERICA'S SEXUAL HEALTH?}

doi:10.1136/sextrans-2011-050108.576

C Mettenbrink, R Cornelis. Denver Public Health Department,Denver USA

Background The implementation of healthcare reform in the U.S has led to a debate on whether publicly-funded STI clinics will continue to be necessary. A large proportion of STIs are reported from non-STI clinic providers and this proportion will grow with universal access to care. However, STI clinics serve vulnerable populations such as men who have sex with men (MSM) and racial/ ethnic minorities who have no other access to STI care. In addition, many prefer the confidentiality and expertise of STI clinics. We studied characteristics of patients reported with gonorrhoea in the City and County of Denver and compared those reported from the Denver Metro Health (STI) clinic (DMHC) with those reported from elsewhere.

Methods As part of the CDC-funded STD Surveillance Network (SSuN), DMHC and the Colorado Department of Public Health and Environment (CDPHE) collect additional risk information for gonorrhoea cases residing in the SSuN catchment area. Of all incident gonorrhoea cases within a given month, 40 are randomly selected for an interview by CDPHE staff to collect demographic, treatment, and risk information. All gonorrhoea cases diagnosed between 1 January 2008 and 31 October 2010 were examined.

Results Of 2933 gonorrhoea cases for the 2-year period, 932 (32\%) were diagnosed at DMHC. Cases diagnosed at DMHC were considerably more likely to be male $(74 \%$ vs $38 \% \mathrm{p}<0.0001)$, and tended to be slightly older (mean age 27.0 vs $24.9, p=<0.0001$ ) than cases diagnosed elsewhere. CDPHE successfully contacted $41 \%$ of selected cases for interviews. A significantly higher proportion of cases diagnosed in the STI clinic were MSM compared to cases diagnosed elsewhere $(34.7 \%$ vs $14.5 \%, \mathrm{p} \leq 0.0001)$. No differences were found in terms of race/ethnicity, number of reported sex partners, education level, or previous gonorrhoea infection.

Conclusions In the City and County of Denver, heterosexual men and MSM are more likely to be diagnosed with gonorrhoea in the STI clinic than women. The successful transition of STI services to non-STI healthcare systems in the context of healthcare reform, will not only depend on the STI expertise in those settings, but in large part on a shift in health seeking behaviours among men, many of whom may be considered core STI transmitters. The continued need for and utilisation of STI clinics in countries with long-standing universal access to care, should give us pause in abandoning our STI clinical system to soon.

\section{P5-S6.21 ACYCLOVIR FOR MANAGING GENITAL ULCER DISEASE IN SOUTH AFRICA: EXPERIENCES OF GUIDELINE INTRODUCTION, IMPLEMENTATION AND UPTAKE}

doi:10.1136/sextrans-2011-050108.577

${ }^{1} \mathrm{D}$ Emge, ${ }^{2} \mathrm{~S}$ Delany-Moretlwe, ${ }^{3} \mathrm{P}$ Mayaud. 'London School of Hygiene \& Tropical Medicine and Reproductive Health \& HIV Research Unit, University of the Witwatersrand, Bay City, USA; ${ }^{2}$ Reproductive Health \& HIV Research Unit, University of the Witwatersrand, Hillbrow, Johannesburg, South Africa; ${ }^{3}$ London School of Hygiene $g$ Tropical Medicine, London, UK

Background Herpes simplex virus type 2 (HSV-2) is the leading cause of genital ulcer disease (GUD) worldwide. Treatment with acyclovir has been shown to shorten GUD duration, decrease plasma and genital HIV RNA levels and slow HIV disease progression in several trials. In South Africa, STI management guidelines were revised in 2008 to include acyclovir as first-line GUD therapy. However, acyclovir access appeared to be unequal across various settings. This study attempted to understand acyclovir access in South Africa from both a supply and demand perspective.

Methods The study was cross-sectional and primarily qualitative. Three groups of participants were recruited to reflect acyclovir access and use: policymaker key informants, healthcare providers at public primary healthcare facilities and ART initiation sites in greater Johannesburg, and former HSV-2 treatment trial participants (both genders, HIV-positive and -negative). Information was collected following the WHO/Health Action International twostage cluster random selection of health units through standardised questionnaires, complemented by in-depth interviews with selected stakeholders.

Results Acyclovir was documented to be widely available. Challenges to access included the initial policy development and implementation process, staff training, accessibility and availability. The updated guidelines appeared to have been introduced with little high-level co-ordination and minimal staff training, although policymakers and staff thought that HSV-2 treatment was both efficacious and useful. Clinics did not experience drug stock outs. The demand for acyclovir appeared to be poor and influenced by limited knowledge of HSV-2 by the general public, negative perceptions associated with HSV-2 infection, adverse logistical factors like long waiting times at clinics and negative experiences with staff at clinics.

Conclusions The South African guidelines were supported by local research evidence in line with WHO recommendations. Although little attention was paid to how the new guidelines had to be introduced, implemented and monitored, both the supply and prescribers' awareness improved rapidly. Access to acyclovir was undermined by poor demand from patients. Individuals possibly did not see herpes as enough of a problem to seek treatment. To ensure the success of new treatment approaches, it is essential to consider user and well as provider issues.

\section{P5-S6.22 SOCIAL-STRUCTURAL FACTORS ASSOCIATED WITH SUPPORTIVE SERVICE USE IN A COHORT OF HIV-POSITIVE INDIVIDUALS ON ARV THERAPY IN BRITISH COLUMBIA, CANADA}

doi:10.1136/sextrans-2011-050108.578

${ }^{1} \mathrm{~N}$ O'Brien, ${ }^{1} \mathrm{~A}$ K Palmer, ${ }^{1} \mathrm{~W}$ Zhang, ${ }^{1} \mathrm{~W}$ Michelow, ${ }^{1} \mathrm{~A}$ Shen, ${ }^{2} \mathrm{E}$ Roth, ${ }^{3} \mathrm{C} \mathrm{L}$ Rhodes, ${ }^{1} \mathrm{~J} S \mathrm{G}$ Montaner, ${ }^{1} \mathrm{R}$ S Hogg. ${ }^{1} B C$-Centre for Excellence in HIVIAIDS, Vancouver, Canada; ${ }^{2}$ University of Victoria, Canada; ${ }^{3}$ University of Waterloo, Canada

Introduction Medical services are seldom the only assistance required for HIV-positive individuals to lead longer, healthier lives. The clinical complexities of HIV infection and the multiple needs of people living with HIV often require additional assistance to ensure 